\title{
ORIGINAL ARTICLE \\ The physiological cost index of walking with mechanical and powered gait orthosis in patients with spinal cord injury
}

\author{
M Arazpour ${ }^{1}$, MA Bani ${ }^{1}$, SW Hutchins ${ }^{2}$ and RK Jones ${ }^{2}$
}

Objectives: Mechanical orthoses, such as the hip knee ankle foot orthosis (HKAFO) and the isocentric reciprocating gait orthosis (IRGO), are both used for walking in spinal cord injury (SCI) patients. The aim of this study was to analyze the energy expenditure during walking with these orthoses compared with a powered gait orthosis (PGO) in patients with SCl.

Methods: Five patients with $\mathrm{SCl}$ who were experienced users of HKAFOs participated in this study. Subjects were also fitted with an IRGO and PGO and underwent a specific gait training program. Patients walked along a flat walkway using the three types of orthosis at their self-selected walking speed. A stop watch and a polar heart rate monitor were used to measure the speed of walking and heart rate.

Results: Walking speed, the distance walked and the physiological cost index (PCI) all improved with both the new PGO and the IRGO as compared with the HKAFO.

Conclusions: A PGO can improve walking speed and the distance walked and reduce the $\mathrm{PCl}$ of walking as compared with mechanical orthoses, probably due to the activated movements of the lower limb joints.

Spinal Cord (2013) 51, 356-359; doi:10.1038/sc.2012.162; published online 18 December 2012

Keywords: reciprocating orthosis; powered gait orthosis; spinal cord injury; walking; physiological cost index

\section{INTRODUCTION}

Standing and walking can help to prevent contractures of the lower limb joints, as well as osteoporosis, spasticity, bed sores and edema in patients with spinal cord injury (SCI). ${ }^{1}$ Various types of orthosis have been developed for this patient group to enable ambulation. The hip knee ankle foot orthosis (HKAFO) has been traditionally used, but this has the disadvantage that it is heavy and provides an inefficient gait. $^{2}$ The reciprocating gait orthosis (RGO) incorporates a mechanism, which allows hip flexion of one side to cause hip extension on the contra-lateral side to facilitate a reciprocating walking pattern; albeit with the knees and ankles locked in position. ${ }^{3}$

However, despite the positive effect of RGOs, their improvement on the temporal spatial parameters and the kinematics of walking for SCI subjects is still limited. ${ }^{3,4}$ Previous studies have demonstrated that walking with an RGO is exhausting for SCI patients, ${ }^{5}$ and rejection of this type of orthosis has been reported as being between 46 and $56 \%$ in patients with SCI. ${ }^{6,7}$ High loads on the upper limb joints and high levels of energy consumption have been reported as the main reasons for this rejection. ${ }^{8,9}$ The use of externally-applied power to specific orthotic joints within devices to assist movement and ambulation has therefore been proposed as an alternative approach in walking and reduced energy consumption in patients with SCI. ${ }^{10,11}$

Different types of powered orthosis have been developed in recent years using pneumatic, hydraulic and electrical actuators. Many studies have been performed to compare the efficacy of such orthoses on the reduction of energy consumption between non-powered orthosis designs, ${ }^{3,4,8}$ but comparison of this parameter between mechanical and powered orthoses has not been extensively evaluated.

The purpose of this study was to analyze the effect of a new powered gait orthosis (PGO) on energy consumption and to compare the energy cost of walking between three different orthosis designs, an $\mathrm{HKAFO}$, an isocentric reciprocating gait orthosis (IRGO) and a new PGO experienced by SCI patients following a formal training period with each orthosis.

\section{MATERIALS AND METHODS}

Patients

Five subjects (two females, three males, $26.8 \pm 2.94$ years, $167 \pm 6 \mathrm{~cm}$ height, $58.2 \pm 4.86 \mathrm{~kg}$ weight and $24 \pm 17$ months post injury time) with $\mathrm{T}_{6}-\mathrm{T}_{12}$ spinal cord lesions participated in the study (Table 1). The patients were characterized by a traumatic SCI with scale B (four patients) and A (one patient) according to American Spinal Injury Association score. They had previous experience of walking with a HKAFO but no experience of walking and standing with an IRGO or PGO. The inclusion criteria included no evidence of cardiovascular or pulmonary diseases, contractures, severe spasticity, obesity or asymmetric hip positions. Informed consent for the study obtained from SCI patients. The ethics committee of University of Social Welfare and Rehabilitation Science approved performance of this study.

\section{Orthosis considerations}

An IRGO (Ortho System in Motion Co., Kirchheim, Germany) was used in this study. The HKAFO consisted of two long leg braces, which were connected together with a pelvic band and a lumbo-sacral orthosis portion. The PGO incorporated synchronized active motion in both hip and knee joints, by using

${ }^{1}$ Department of Orthotics and Prosthetics, University of Social Welfare and Rehabilitation Science, Tehran, Iran and ${ }^{2}$ HSCR, Faculty of Health and Social Care, University of Salford, Salford, UK

Correspondence: Dr MA Bani, Department of Orthotics and Prosthetics, University of Social Welfare and Rehabilitation Science, Kodakyar Street, Daneshjo Boulevard, Evin, Tehran 1985713834, Iran.

E-mail: M.ahmadi_bani@yahoo.com

Received 16 August 2012; revised 25 October 2012; accepted 9 November 2012; published online 18 December 2012 
three battery-driven electrical actuators acting on the hip and knee joints. Design and simulation of this orthosis has been reported previously on a single SCI patient. ${ }^{12}$ Speed of walking, cadence and step length was increased and vertical and lateral compensatory motions were reduced when the patient walked with it donned. ${ }^{11}$ The volunteer subjects used the same HKAFO that they had worn previously. In this study, the ankle foot orthoses (AFOs) incorporated in the IRGO and the PGO were custom molded using plaster of Paris casts of the lower limbs for each volunteer subject, and the superstructures of both these orthoses and the superstructure for each was adjusted and adapted to fit each patient. Figure 1 shows the three types of orthosis used in this study. For the PGO, the actuators were powered by a battery power pack placed in a haversack, which the volunteer subjects wore during the walking trials and training sessions. A connection lead was used to connect to the power supply to the actuators.

\section{Training program}

All patients attended a walking and standing program with the IRGO and the PGO. A period of 1-month resting time was taken between each gait training program with each orthosis to limit any inter-training effect. Subjects trained for 8 -weeks of gait training after construction of each orthosis. There were three sessions per week of $2 \mathrm{~h}$ duration with each orthosis. The gait training program also consisted of passive stretching of the lower extremities, upper limb strengthening, balancing with the orthosis, plus standing and walking.

\section{Experimental approach}

A stop watch was used to measure the speed of walking. Subjects were asked to walk using three types of orthosis in random order, along a predetermined 40-m rectangular walkway at their self-selected speed. The interval between the starting and stopping time was used to calculate the walking speeds.

\section{Table 1 Demographics of patients included in this study}

\begin{tabular}{llcccccc}
\hline Patient Gender & $\begin{array}{c}\text { Age } \\
\text { (years) }\end{array}$ & $\begin{array}{c}\text { Height } \\
\text { (cm) }\end{array}$ & $\begin{array}{c}\text { Weight } \\
(\mathrm{kg})\end{array}$ & $\begin{array}{c}\text { Level of } \\
\text { injury }\end{array}$ & $\begin{array}{c}\text { ASIA } \\
\text { score }\end{array}$ & $\begin{array}{c}\text { Time since injury } \\
\text { (months) }\end{array}$ \\
\hline 1 & Male & 29.0 & 165.0 & 53.0 & T12 & B & 32.0 \\
2 & Female & 22.0 & 165.0 & 54.0 & T8 & B & 51.0 \\
3 & Male & 26.0 & 178.0 & 65.0 & T10 & A & 15.0 \\
4 & Female & 29.0 & 160.0 & 59.0 & T6 & B & 9.0 \\
5 & Male & 28.0 & 170 & 60.0 & T8 & B & 14.0 \\
\hline
\end{tabular}

Abbreviation: ASIA, American Spinal Injury Association.
A polar heart rate monitor was used to obtain information to evaluate the physiological cost index (PCI). ${ }^{13}$ To calculate the PCI using this approach, the heart rate at steady state walking (HRss) and the heart rate at rest (HRar) were measured, and the following formula used to measure the PCI:

PCI $($ beats $/ \mathrm{m})=($ HRss - HRar $) / \mathrm{V}$

The three types of orthosis were used randomly. To obtain a baseline level of PCI, subjects sat for $10 \mathrm{~min}$ before any measurement. The method of PCI measurement, included $5 \mathrm{~min}$ complete rest in sitting position, $5 \mathrm{~min}$ in standing position, walking with the orthosis for $6 \mathrm{~min}$ at their self-selected speed of walking and a final 2 min of rest in the seated position. During all these activities (that is, during resting, standing and walking), the heart rate was measured every $15 \mathrm{~s}$. After $10 \mathrm{~min}$ of rest in the sitting position with the next orthosis, the next evaluation and measurement was started. The mean of heart rate during the final $2 \mathrm{~min}$ of rest in the sitting position was considered as HRar and average heart rate in 6 min walking with orthosis was noted as HRss.

\section{Statistical analysis}

The collected data for the three conditions of walking with orthoses were averaged for the calculation of speed, distance of walking and PCI. A one way analysis of variance was used for analysis. SPSS 16 (SPSS Inc, Chicago, IL, USA) was used for data analysis. The significant level considered was $\alpha=$ QUOTE . Using a retrospective power analysis, the following results were obtained: speed-1000, distance -0.946 and PCI -0.997 . The confidence intervals for the analysis were as follows: speed-PGO versus IRGO $=(5.413-$ 9.267), $\mathrm{PGO}$ versus $\mathrm{HKAFO}=(4.690-7.110)$; $\mathrm{PCI}-\mathrm{PGO}$ versus $\mathrm{IRGO}=($ -1.325 to -0.700$)$, PGO versus $\mathrm{HKAFO}=(-1.481$ to -0.626$)$; distance PGO versus $\mathrm{IRGO}=(5.723-41.477)$ and $\mathrm{PGO}$ versus $\mathrm{HKAFO}=(12.834-$ 46.766).

\section{RESULTS}

Table 2 demonstrates the mean \pm s.d. of speed of walking, distance and PCI during the three orthotics walking conditions.

In speed of walking there was a significant difference between orthotic walking conditions $(P$ value $<0.001)$. Walking with PGO improved the speed of walking as compared with the HKAFO ( $P$ value $<0.001)$ and the IRGO $(P$ value $=0.001)$, but there was no significant difference between the HKAFO and IRGO in the speed of walking $(P$ value $=0.748)$ (Table 3$)$.

When considering the distance walked, there was a significant difference between orthotic walking conditions $(P$ value $=0.006)$. Wearing the PGO improved the distance walked as compared with the
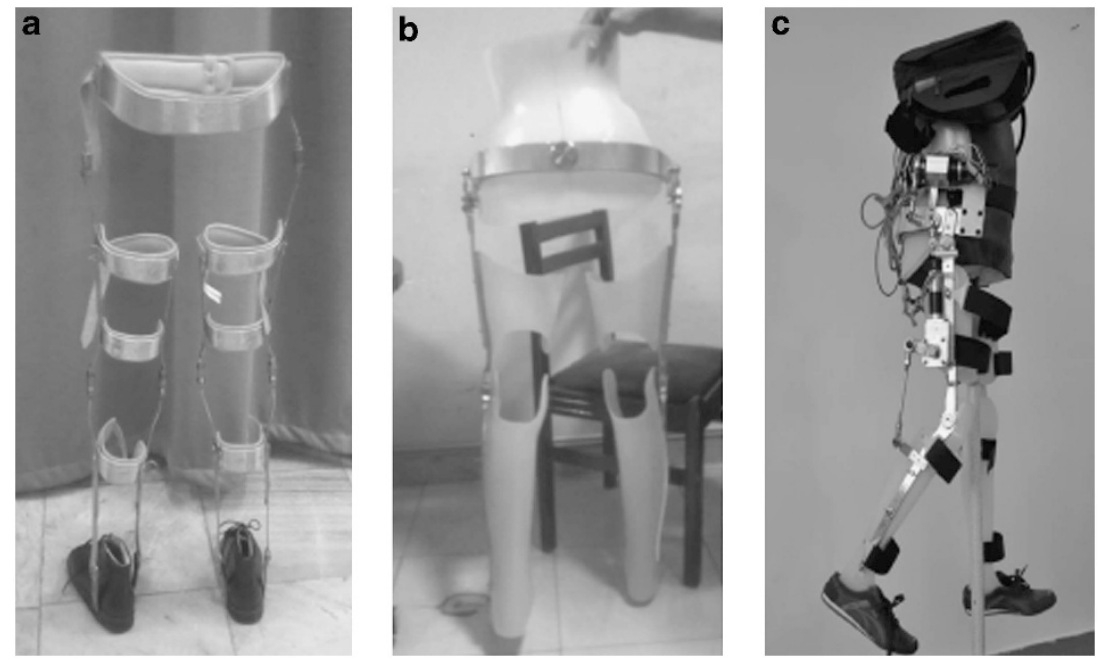

Figure 1 Orthoses used in this study: (a) HKAFO, (b) IRGO and (c) PGO. 
Table 2 Mean \pm s.d. and comparison of speed of walking, distance and $\mathrm{PCl}$ during the three orthotic conditions

\begin{tabular}{lccc}
\hline & $\begin{array}{c}\text { Speed of walking } \\
(m \text { per } \text { min) }\end{array}$ & $\begin{array}{c}\text { Distance } \\
\text { walked }(m)\end{array}$ & $\begin{array}{c}\text { PCl } \\
\text { (beats per min) }\end{array}$ \\
\hline Walking with HKAFO & $13.84 \pm 1.85$ & $90.20 \pm 10.63$ & $1.97 \pm 0.17$ \\
Walking with IRGO & $15.28 \pm 2.02$ & $96.40 \pm 13.35$ & $1.93 \pm 0.40$ \\
Walking with PGO & $21.18 \pm 1.75$ & $120 \pm 12.98$ & $0.92 \pm 0.25$ \\
$P$ value & 0.000 & 0.006 & 0.000 \\
\hline
\end{tabular}

Abbreviations: HKAFO, knee ankle foot orthosis; IRGO, isocentric reciprocating gait orthosis; $\mathrm{PCl}$, physiological cost index; $\mathrm{PGO}$, powered gait orthosis.

Table 3 Between-group comparison of speed of walking, distance and $\mathrm{PCl}$ in the three orthotic conditions

\begin{tabular}{lccc}
\hline & $\begin{array}{c}\text { Speed of } \\
\text { walking } \\
\text { (m per min) }\end{array}$ & $\begin{array}{c}\text { Distance } \\
\text { walked } \\
(\mathrm{m})\end{array}$ & $\begin{array}{c}\mathrm{PCl} \\
\text { (beats per } \\
\text { min) }\end{array}$ \\
\hline Comparison between HKAFO and IRGO & 0.748 & 1.000 & 1.000 \\
Comparison between HKAFO and PGO & 0.000 & 0.008 & 0.000 \\
Comparison between PGO and IRGO & 0.001 & 0.032 & 0.000 \\
\hline
\end{tabular}

Abbreviations: HKAFO, knee ankle foot orthosis; IRGO, isocentric reciprocating gait orthosis;

$\mathrm{PCl}$, physiological cost index; PGO, powered gait orthosis.

HKAFO $(P$ value $=0.008)$ and the IRGO $(P$ value $=0.032)$, but again there were no significant difference between the HKAFO and IRGO in this parameter $(P$ value $=1.000)($ Table 3$)$.

In PCI there was a significant difference between orthotic walking conditions $(P$ value $=0.006)$. The PGO also improved the parameters of walking speed and the distance walked compared to that demonstrated by the HKAFO ( $P$ value $<0.001)$ and IRGO ( $P$ value $<0.001)$. There was no significant difference between HKAFO and IRGO in energy consumption $(P$ value $=1.000)($ Table 3$)$.

\section{DISCUSSION}

SCI patients with high thoracic lesions have variation in the amount of paralysis experienced in the trunk and lower limb muscles. ${ }^{13}$ On the basis of their level of paralysis, the patients who volunteered for this study had routinely used a HKAFO to ambulate and perform activities of daily living. Following attendance for the 8 weeks of gait training period with the IRGO, the results demonstrated an improved speed of walking and also a reduction in energy expenditure as compared with walking with the HKAFO. Gait training with the PGO had a more significant effect in these two parameters as compared with the HKAFO.

Measurement of heart rate was used to evaluate the PCI of walking with the three orthoses and has been used in previous studies. ${ }^{3}$ When walking with the HKAFO, subjects had a high energy expenditure, confirming the results of a previous study in this field. ${ }^{3}$ Use of the IRGO improved the mean of PCI in these patients due to the reciprocal link between to side of orthosis. A similar result has been reported in previous study. ${ }^{3}$ Walking with the PGO improved all parameters as compared with the other two devices. Using electrically powered actuators in the new PGO acting on the hip and knee joints facilitated motion in these joints. There was no significant difference between the two non-powered orthoses in this parameter.
Both the speed and distance walked with the HKAFO and IRGO was low as compared with walking with the PGO. The limited ranges of motion associated with paralysis of muscle in the lower limb can be the cause of low speed of walking when using a HKAFO. Typical speeds of walking have been reported as being between $0.2-0.3 \mathrm{~m} \mathrm{~s}^{-1}$ with an energy consumption of $1.0 \mathrm{ml} \mathrm{kg}^{-1} \mathrm{~m}^{-1}$ with RGOs in SCI patents. ${ }^{14,15}$ However, in healthy subjects, the average gait velocity and energy consumption have been reported as being $1.28 \mathrm{~m} \mathrm{~s}^{-1}$ and $0.176 \mathrm{ml} \mathrm{kg}^{-1} \mathrm{~m}^{-1}$, respectively. ${ }^{16}$ Leung et al. ${ }^{3}$ reported that the speed and distance of walking improved when using the IRGO as compared with an HKAFO. Harvey et al. ${ }^{17}$ demonstrated that using the IRGO improved speed of walking as compared with the Walkabout orthosis. Arazpour et al. ${ }^{10,11}$ reported the using a powered hip orthosis and also a RGO improved kinematics and temporal spatial parameters as compared with the IRGO in a SCI patient. Kawashima et al. ${ }^{18}$ demonstrated that using the weight bearing control orthosis reduced energy consumption and improved speed of walking in paraplegic patients.

Using a PGO has been shown to be an effective approach in walking and has the potential to reduce energy consumption in SCI patient. ${ }^{10,11}$ As compared with other type of hybrid systems such as functional electrical stimulation, ${ }^{15}$ this system is considered effective according to the muscle fatigue previously demonstrated when using functional electrical stimulation. Although this comparison was not performed in this study, a comparison between the two approaches is proposed for further study.

The weights of the orthoses tested in this study were 5.6, 6.7 and $10.1 \mathrm{~kg}$, respectively, for the HKAFO, IRGO and PGO. However, the weight of the PGO had little effect on the gait and energy consumption in walking, by the SCI patients in this study.

Gait training has a positive effect on walking in SCI patients. ${ }^{19}$ However, only 8 weeks of gait training was used in this study, and a further study with long-term use of the orthosis follow gait training is proposed as further design of study.

\section{Clinical relevance}

Powered gait orthoses are a new generation of assistive devices as a method in rehabilitation of walking and standing in patients with SCI. According to the improvement of energy consumption of this type of orthosis in this study, they may prove to provide more efficiently walking in patients with SCI as compared with mechanical orthosis.

\section{CONCLUSIONS}

PGO facilitate standing and walking similar to the IRGO and HKAFO. In this study we observed that gait training with new PGO improved the speed of walking as compared to IRGO and HKAFO. A similar result was shown in energy consumption. Contraction of muscles in the trunk and upper limb for ambulation and high loading through the upper limbs may be a cause of the high rate of energy expenditure when using an HKAFO or an IRGO compared to the activated movement of the lower limbs produced by a PGO. In conclusion, patients with SCI walk faster and more efficiently using a PGO and its use should therefore be proposed for the SCI patient.

\section{DATA ARCHIVING}

There were no data to deposit.

\section{CONFLICT OF INTEREST}

The authors declare no conflict of interest. 
1 Walter J, Sola P, Sacks J, Lucero Y, Langbein E, Weaver F. Indications for a home standing program for individuals with spinal cord injury. J Spinal Cord Med 1999; 22: 152.

2 Moore P, Stallard J. A clinical review of adult paraplegic patients with complete lesions using the ORLAU Parawalker. Spinal Cord 1991; 29: 191-196.

3 Leung AKL, Wong AFY, Wong ECW, Hutchins SW. The physiological cost index of walking with an isocentric reciprocating gait orthosis among patients with T12-L1 spinal cord injury. Prosthet Orthot Int 2009; 33: 61-68.

4 Winchester P, Carollo J, Parekh R, Lutz L, Aston Jr J. A comparison of paraplegic gait performance using two types of reciprocating gait orthoses. Prosthet Orthot Int 1993; 17: 101-106.

5 Bernardi M, Canale I, Castellano V, Di Filippo L, Felici F, Marchetti M. The efficiency of walking of paraplegic patients using a reciprocating gait orthosis. Spinal Cord 1995; 33: 409-415.

6 Franceschini M, Baratta S, Zampolini M, Loria D, Lotta S. Reciprocating gait orthoses: a multicenter study of their use by spinal cord injured patients. Arch Phys Med Rehabil 1997; 78: 582-586.

7 Scivoletto G, Petrelli A, Di Lucente L, Giannantoni A, Fuoco U, D'ambrosio F et al. One year follow up of spinal cord injury patients using a reciprocating gait orthosis: preliminary report. Spinal Cord 2000; 38: 555-558.

8 Massucci M, Brunetti G, Piperno R, Betti L, Franceschini M. Walking with the advanced reciprocating gait orthosis (ARGO) in thoracic paraplegic patients: energy expenditure and cardiorespiratory performance. Spinal Cord 1998; 36: 223-227.

9 Jaspers P, Peeraer L, Van Petegem W, Van der Perre G. The use of an advanced reciprocating gait orthosis by paraplegic individuals: a follow-up study. Spinal Cord 1997; 35: 585-589.
10 Arazpour M, Chitsazan A, Hutchins SW, Ghomshe FT, Mousavi ME, Takamjani EE et al. Evaluation of a novel powered hip orthosis for walking by a spinal cord injury patient: a single case study. Prosthet Orthot Int 2012; 36: 105-112.

11 Arazpour M, Chitsazan A, Hutchins SW, Mousavi ME, Takamjani EE, Ghomshe FT et al. Evaluation of a novel powered gait orthosis for walking by a spinal cord injury patient. Prosthet Orthot Int 2012; 36: 239-246.

12 Arazpour M, Chitsazan A, Hutchins SW, Ghomshe FT, Mousavi ME, Takamjani EE et al. Design and simulation of a new powered gait orthosis for paraplegic patients. Prosthet Orthot Int 2012; 36: 125-130.

13 Kawashima N, Taguchi D, Nakazawa K, Akai M. Effect of lesion level on the orthotic gait performance in individuals with complete paraplegia. Spinal Cord 2006; 44: 487-494.

14 Bernardi M, Canale I, Castellano V, Di Filippo L, Felici F, Marchetti M. The efficiency of walking of paraplegic patients using a reciprocating gait orthosis. Paraplegia 1995; 33: 409-415.

15 Hirokawa S, Grimm M. Energy consumption in paraplegic ambulation using the reciprocating gait orthosis and electric stimulation of the thigh muscles. Arch Phys Med Rehabil 1990; 71: 687.

16 Bernardi M, Macaluso A, Sproviero E, Castellano V, Coratella D, Felici F et al. Cost of walking and locomotor impairment. J Electromyography Kinesiol 1999; 9: 149-157.

17 Harvey LA, Smith MB, Davis GM, Engel S. Functional outcomes attained by T9-12 paraplegic patients with the walkabout and the isocentric reciprocal gait orthoses. Arch Phys Med Rehabil 1997; 78: 706-711.

18 Kawashima N, Sone Y, Nakazawa K, Akai M, Yano H. Energy expenditure during walking with weight-bearing control (WBC) orthosis in thoracic level of paraplegic patients. Spinal Cord 2003; 41: 506-510.

19 Ferris DP, Sawicki GS, Domingo AR. Powered lower limb orthoses for gait rehabilitation. Top Spinal Cord Inj Rehabil 2005; 11: 34-49. 